\section{ARTIGO ORIGINAL ORIGINAL ARTICLE}

\title{
Modelo de gerenciamento de projetos aplicado a pesquisa clínica
}

\author{
Project management model for clinical research \\ Angelica Dutra Zanotto', Karen Brasil Ruschel², Carisi Anne Polanczyk \\ DOI: 10.21115/JBES.v10.n3.p232-8
}

\section{RESUMO}

Objetivo: Desenvolver um modelo para o gerenciamento de projetos em centros de pesquisa clínica (CPCS) no cenário público-privado e privado. Métodos: Estudo transversal prospectivo com aplicação de um questionário específico em CPCs no Brasil. Esse instrumento avaliou a aplicação prática das 10 áreas de conhecimento do Project Management Body of Knowledge (PMBOK ${ }^{\circledR}$ ) (gerenciamento da integração, do escopo, do tempo, de custos, de qualidade, de recursos humanos, de comunicações, de riscos, de aquisições e partes interessadas) com relação à prática diária da pesquisa clínica nos CPC do país. Resultados: Dos 195 centros de pesquisa elegíveis, 55 responderam ao questionário completo. O profissional com o cargo de gerente de projetos é inexistente em 37 centros (67,3\%) ativos e não há Escritório de Gerenciamento de Projetos (EGP) em 41 centros (74,5\%). O controle de despesas e receitas é realizado por 50 centros (90,9\%), entretanto $28(50,9 \%)$ realizam avaliação da rentabilidade. Quanto ao gerenciamento da qualidade, 28 (50,9\%) não têm parâmetros de qualidade implantados e 11 (40,7\%) não realizam auditoria interna. Falhas de comunicação estão presentes em 48 centros (87,2\%). A partir da avaliação da aplicação prática das 10 áreas de conhecimento do Guia PMBOK ${ }^{\circledR}$, foi desenvolvido um modelo de gerenciamento de projetos com aplicabilidade aos centros. Conclusões: É possível que os centros tenham melhor definição do escopo de cada projeto, custos e prazos possuam baixa margem de variabilidade, se estabeleça boa comunicação entre as partes envolvidas e que o impacto econômico do gerenciamento possa ser reconhecido.

\section{Keywords:}

health management, knowledge management for health research, academies and institutes

\begin{abstract}
Objective: To develop a model for the management of research projects, in public-private and private scenarios. Methods: Prospective cross-sectional study with the application of a specific questionnaire for research centers in the country. This instrument evaluated the practical application of the 10 areas of Project Management Body of Knowledge (PMBOK ${ }^{\circledR}$ ) (management of integration, scope, time, costs, quality, human resources, communications, risks, acquisitions and stakeholders) of in relation to the daily practice of clinical research in the country's research centers. Results: Of the 195 eligible research centers, 55 centers answered the complete questionnaire. The professional with the position of project manager does not exist in 37 active centers (67.3\%), there is no project management office (PMO) in 41 centers (74.5\%). The control of expenses and revenues is carried out by 50 centers (90.9\%), however, 28 (50.9\%) carry out an evaluation of the profitability. Regarding quality management, 28 (50.9\%) did not have quality parameters implanted and 11 (40.7\%) did not perform internal audit. Communication failures are present in 48 (87.2\%) centers. Based on the evaluation of the practical application of the 10 knowledge areas of the PMBOK ${ }^{\circledR}$ Guide, the project management model was developed with applicability to clinical research centers. Conclusions: It is possible that the centers have a better definition of the scope of each project, the costs and deadlines have a low margin of variability, a good communication between the parties is established and the economic impact of the management can be recognized.
\end{abstract}

\footnotetext{
Recebido em: 21/11/2018. Aprovado para publicação em: 26/12/2018.

1. Programa de Pós-Graduação do Hospital de Clínicas de Porto Alegre, Instituto de Avaliação de Tecnologias em Saúde. 2. Instituto de Avaliação de Tecnologias em Saúde; Programa de Pós-Graduação em Cardiologia e Ciência Cardiovasculares da Universidade Federal do Rio Grande do Sul.

Instituição onde o trabalho foi realizado: Hospital de Clínicas de Porto Alegre.

Autor correspondente: Karen Brasil Ruschel. Instituto de Avaliação de Tecnologias em Saúde. Avenida Ramiro Barcelos, 2350, Porto Alegre, RS, Brasil. CEP: 90035-903. Telefone: +55 (51) 3359-6325. E-mail: karenbruschel@gmail.com
} 


\section{Introdução}

A globalização da pesquisa clínica está fortemente ligada ao processo de internacionalização e terceirização de Pesquisa e Desenvolvimento (P\&D) da indústria farmacêutica e insumos em saúde. Nesse contexto, a escolha pelo local de sua execução envolve considerações sobre custo, recrutamento de pacientes, infraestrutura e ambiente ético-regulatório (Silva et al., 2016). No Brasil, o cenário da pesquisa clínica tem se mostrado favorável e com grande potencial de desenvolvimento. Algumas características contribuem para esse cenário, como o país ter grande massa populacional com alta incidência de doenças, heterogeneidade, condições climáticas distintas, associadas a médicos capacitados e adequada estrutura de centros de pesquisa clínica (CPCS) ativos (Laboratórios Pfizer, 2017). Entretanto, apesar do crescimento nos últimos anos, permanecem escassos os CPCs com expressivo recrutamento de pacientes e entregas de qualidade em cenário nacional, tornando um desafio se manter competitivo em um cenário internacional ágil e estruturado para essas demandas.

A complexidade de gerenciar um CPC com objetivos e metas voltados para a otimização dos custos, prazos, qualidade, desempenho e benefícios esperados deve-se ao gerenciamento de processos únicos e descontínuos.

A criação de projetos envolve precisamente o esforço em cumprir objetivos apesar dos imprevistos, do incalculável, do conturbado e do acidental (Sabbag, 2013). Seja qual for o projeto, a aplicação de processos de gerenciamento é reconhecida como fator de sucesso.

O conhecimento da forma como os centros de pesquisa executam seus estudos clínicos no âmbito gerencial possibilita reconhecer as práticas de sucesso implementadas e propicia o avanço do conhecimento a práticas inovadoras e conceituadas de gerenciamento de projetos. Devido às diferentes áreas de atuação dos centros, em que se correlacionam diferentes expertises para a formação das equipes, é comum estas serem formadas por profissionais das diversas especialidades em saúde, que muitas vezes desconhecem ou possuem dificuldades de aplicar técnicas e ferramentas para o gerenciamento do portfólio de estudos que o centro conduz.

Como na maior parcela dos CPCs do Brasil ainda não está instituída uma cultura organizacional de gerenciamento de projetos baseado em um modelo de gestão, associado à escassa presença do profissional gerente de pesquisa, administrador de pesquisa ou gerente de projetos, a criação de um modelo de gerenciamento voltado exclusivamente à pesquisa clínica, usando o guia metodológico do Project Management Body of Knowlegde (PMBOK ${ }^{\oplus}$ ) como "padrão-ouro", resulta em um produto prático e aplicável pelos profissionais em atividade na pesquisa clínica do país. Até o momento, não se conhece um modelo padronizado para o gerenciamento dos CPCs de uso compartilhado no país.
Este estudo objetivou desenvolver um modelo para o gerenciamento de projetos de pesquisa em CPCs do cenário público-privado e privado do Brasil, com foco em entregas, eficiência e sustentabilidade.

\section{Métodos}

Estudo de caráter transversal prospectivo com aplicação de um questionário estruturado de pesquisa para CPCs no país.

\section{População-alvo}

Foram selecionados CPCs no país, independentemente das áreas de atuação, considerando ao menos um centro de cada região do Brasil. Entre os centros identificados, foram selecionados os menores Núcleos de Pesquisa com atividade específica e atividades gerenciais individualizadas e autônomas.

A amostragem dos centros foi por conveniência a partir dos cadastros de centro de pesquisa disponíveis na Empresa Brasileira de Serviços Hospitalares - Ebserh (37 contatos), Rede Nacional de Pesquisa Clínica - RNPC (40 contatos), Sociedade Brasileira de Profissionais em Pesquisa Clínica - SBPPC (23 contatos), Hospital de Clínicas de Porto Alegre (30 contatos), Registro Brasileiro de Ensaios Clínicos - Rebec (58 contatos), contatos aleatórios por meio de busca na internet e networking (22 contatos). Totalizaram-se 210 contatos.

Critérios de inclusão:

- Estar em atividade de pesquisa clínica no mínimo há dois anos;

- Apresentar um ou mais estudos ativos no momento do envio do questionário;

- Estar vinculado a uma instituição hospitalar.

Critérios de exclusão:

- Não responder ao questionário no período estipulado para a coleta de dados;

- Questionários com $\leq 50 \%$ de preenchimento.

\section{Coleta de dados}

A coleta de dados foi realizada por meio do envio de um questionário específico durante os meses de março - agosto de 2017, aos CPC (Figura 1). O questionário aborda as 10 áreas de conhecimento do Guia PMBOK (PMI, 2013), com relação à prática diária da pesquisa clínica (gerenciamento da integração, do escopo, do tempo, de custos, de qualidade, de recursos humanos, de comunicações, de riscos, de aquisições e partes interessadas).

A definição básica das 10 áreas de conhecimento do Guia PMBOK${ }^{\oplus}$ foi transportada para a aplicabilidade e a rotina diária da pesquisa clínica.

O questionário foi composto por 40 questões de múltipla escolha, respondidas de forma anônima, via plataforma eletrônica Google Formulários ${ }^{\circledR}$. 


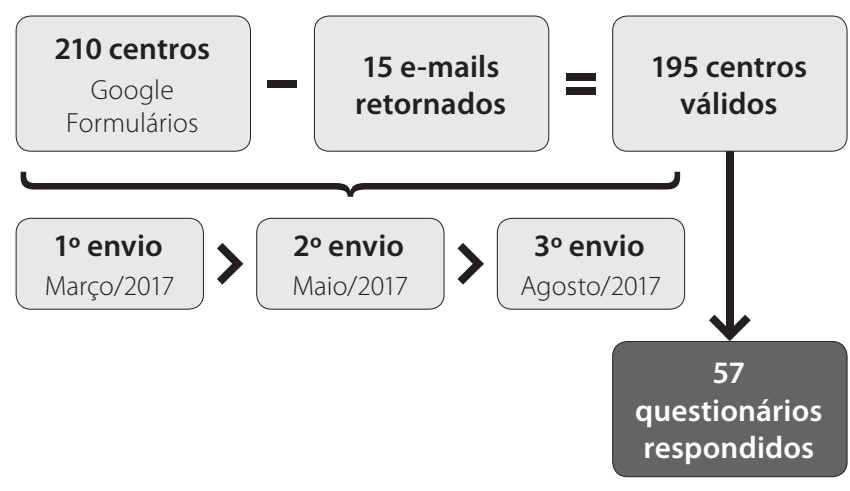

Figura 1. Fluxo de envio do questionário de pesquisa.

A primeira sessão de perguntas do questionário se destina à identificação e à caracterização do centro e do participante que respondeu ao instrumento, sendo composta por oito questões.

As próximas sessões, compostas no total de 21 questões, foram de perguntas focadas em cada tipo de gerenciamento das áreas de conhecimento do PMBOK ${ }^{\oplus}$.

No gerenciamento das aquisições, as perguntas foram relacionadas à origem dos serviços de gestão administrativa e financeira utilizados pelo centro, visando identificar os processos para aquisição de bens, serviços ou resultados com o apoio de terceiros. A intermediação para centros de pesquisa pode ser realizada por fundação de apoio, associação, instituição hospitalar, interveniente administrativo (terceirizado) ou por meio de infraestrutura própria de gestão administrativa e financeira. O maior número de questões do questionário foi para esse tipo de gerenciamento (nove questões) visando conhecer de forma detalhada a realidade de cada centro.

E a última sessão do questionário, com duas questões, foi específica para conhecer quais centros possuem Escritório de Gerenciamento de Projetos (EGP), que auxilia na padronização de processos relacionados à governança dos projetos.

O tempo estimado de resposta foi de 10 minutos.

O questionário foi submetido à fase piloto, com revisão das questões por dois especialistas independentes na área de pesquisa clínica.

Os dados foram recebidos até 31 de agosto de 2017 e a coleta deu-se por encerrada nessa data.

\section{Análise de dados}

Os dados foram tabulados no Microsoft Excel a partir da extração deles do Google Formulários ${ }^{\oplus}$ e por meio da estatística descritiva. Para a criação do modelo de gerenciamento foi utilizado o PMBOK ${ }^{\circledR} 5^{a}$ edição como guia de referência metodológico (PMI, 2013).

\section{Aspectos éticos}

Projeto aprovado pelo Comitê de Ética em Pesquisa do Hospital de Clínicas de Porto Alegre CEP/HCPA (CAAE 62798816.4.0000.5327). O consentimento dos participantes em compartilhar informações do seu CPC, ao responder o questionário distribuído de forma eletrônica, realizou-se quando o participante respondeu ao questionário de forma on-line.

\section{Resultado}

A taxa de resposta obtida com a aplicação do questionário de pesquisa foi de $27,14 \%$. As respostas dos 55 CPCs são correspondentes à participação de 17 estados brasileiros. Foram excluídos da análise dos resultados dois centros de pesquisa. Um dos centros não estava com estudos ativos no momento do preenchimento do questionário e o outro não atuava como CPC.

Os centros de pesquisa vinculados a instituições públicas tiveram maior participação nos resultados com 35 (61,40\%) centros, o setor privado com 14 (24,60\%) centros e o público-privado com 8 (14\%). Os principais respondentes foram coordenadores(as) de estudos de pesquisa clínica, 19 (33,3\%) (Tabela 1).

Uma das principais entregas ao patrocinador dos estudos clínicos é o recrutamento de pacientes. Questionou-se aos centros se havia treinamento para atingir esse resultado. Logo, 51 (92,7\%) dos 55 centros que responderam confirmam que realizam essa atividade continuamente.

Em relação ao gerenciamento do tempo, as etapas de pré-aprovação do estudo, que em grande parte dependem de aprovações de órgãos externos, são de conhecimento de $52(94,5 \%)$ centros de pesquisa.

Por se tratar da normativa regulatória da Anvisa para a pesquisa clínica no país, a Instrução Normativa no 4/2009 (Guia de Inspeção em Boas Práticas Clínicas) foi o principal

Tabela 1. Perfil dos centros de pesquisa no país participantes da pesquisa

\begin{tabular}{lc}
\hline Perfil dos centros participantes & $\begin{array}{c}\text { Taxa de resposta } \\
\mathbf{n}(\%)\end{array}$ \\
\hline $\begin{array}{l}\text { Profissional com o cargo de gerente } \\
\text { de projetos inexistente }\end{array}$ & $37(67,3 \%)$ \\
\hline $\begin{array}{l}\text { Não há Escritório de Gerenciamento } \\
\text { de Projetos (EGP) }\end{array}$ & $41(74,5 \%)$ \\
\hline Controle de despesas e receitas realizado & $50(90,9 \%)$ \\
\hline Realizam avaliação da rentabilidade & $28(50,9 \%)$ \\
\hline $\begin{array}{l}\text { Não possuem parâmetros de } \\
\text { qualidade implantado }\end{array}$ & $28(50,9 \%)$ \\
\hline Não realizam auditoria interna & $11(40,7 \%)$ \\
\hline Falhas de comunicação estão presentes & $48(87,2 \%)$ \\
\hline
\end{tabular}

Fonte: Dados da pesquisa. Elaboração própria. 
parâmetro de qualidade apontado como prática de qualidade nos centros, ao serem questionados sobre quais são os parâmetros de qualidade estabelecidos.

Com o propósito de conhecer sobre a auditoria interna realizada nos centros, foi questionado quem a realiza. Em 10 $(62,5 \%)$ centros, a auditoria interna é realizada por algum integrante da equipe, designado para essa função; 14 (87,5\%) centros fazem checking list de atividades planejadas e realizadas e registram em ata as reuniões da equipe para planejar, realizar e controlar a garantia da qualidade.

Em relação ao uso de interveniente administrativo para gerenciar os pagamentos do estudo, o questionário demonstrou que 21 (38,2\%) centros utilizam um interveniente administrativo (terceirizado) como fonte responsável pela remuneração da equipe de pesquisa. As fundações de apoio a projetos correspondem a $19(34,5 \%)$ respostas, sendo a segunda principal responsável pelo gerenciamento da remuneração da equipe.

Quanto à comunicação entre as partes interessadas, 45 $(81,8 \%)$ centros afirmaram que possuem uma pessoa da equipe designada para se comunicar formalmente com o Comitê de Ética em Pesquisa (CEP), a Comissão Nacional de Ética em Pesquisa (Conep), entre outras entidades regulatórias. Em relação à comunicação interna, $34(61,8 \%)$ responderam que a equipe realiza reuniões presenciais de acompanhamento dos estudos com a equipe do centro; desses, $11(32,4 \%)$ responderam que as reuniões acontecem somente em momentos pontuais, sem frequência predeterminada.

Buscando identificar riscos que podem afetar a sustentabilidade dos centros, foi questionado sobre quantos projetos de pesquisa ativos o centro possui. Centros com número mínimo de 1 a 5 estudos ativos correspondem a $24(43,6 \%)$ e acima de 15 projetos ativos correspondem a 18 (32,7\%).

Em relação à gestão administrativa e financeira sobre serviços com terceiros, 52 (94,5\%) centros de pesquisa responderam que realizam análise de orçamento (budget) prévia de seus estudos clínicos antes da assinatura de contrato, sendo essa atividade realizada pela própria infraestrutura em 29 $(55,8 \%)$ centros.

Sobre o acesso do investigador ao demonstrativo dos créditos e despesas do(s) projeto(s), 50 (90,9\%) centros afirmam ter disponíveis esses dados para visualização.

Sobre se há uma taxa fixa por parte da instituição para o desenvolvimento de projeto(s) de pesquisa, tal como overhead ou taxa, 43 (78,2\%) afirmaram que há taxa para essa atividade. Dos 43 centros, 15 (34,9\%) deles informaram que a taxa aplicada para o uso do serviço é de 10\% a 15\%.

Identifica-se como uma das principais partes interessadas, em estudos de pesquisa clínica, o pesquisador e/ou investigador principal. Quanto à participação no resultado financeiro global dos estudos, 36 (65,5\%) centros afirmam que existe essa prática gerencial no centro.
Ao questionar esses 36 centros sobre a frequência que isso ocorre, $12(33,3 \%)$ afirmaram que trimestralmente o repasse do resultado financeiro do centro ao pesquisador/investigador principal é realizado.

Encerrando o inquérito, perguntou-se sobre a administração dos centros, se possuem um EGP, vinculado à instituição hospitalar. E 41 (74,5\%) informaram que não possuem um EGP ativo. Somente aos 14 (25,5\%) centros que já possuem um EGP ativo foi questionado o seu grau de satisfação em relação ao EGP. No retorno de 7 (50\%) centros, eles avaliaram com nota 8, em uma escala com variação de 1 a 10.

A partir da descrição dos resultados, foi desenvolvido um produto com aplicabilidade prática aos CPCs do país. Ele contempla um fluxo com os cinco grupos de processos de gerenciamento de projetos: iniciação, planejamento, execução, monitoramento e controle, e encerramento. Os grupos de processos integram 10 áreas de conhecimento do PMBOK ${ }^{\oplus}$ : Gerenciamento da Integração, Gerenciamento do Escopo, Gerenciamento do Tempo, Gerenciamento dos Custos, Gerenciamento da Qualidade, Gerenciamento de Recursos Humanos, Gerenciamento das Comunicações, Gerenciamento de Riscos, Gerenciamento das Aquisições e Gerenciamento de Stakeholders. Cada área de conhecimento está interligada aos 29 processos relacionados à pesquisa clínica, formando o modelo de gerenciamento aplicado aos CPCs.

No presente artigo apresentaremos o modelo de forma descritiva (Figura 2).

A construção desse fluxo foi consolidada em uma única figura com o propósito de ser visualmente acessível, de fácil manuseio pelos profissionais ligados à gestão da pesquisa clínica e compreensível por meio de processos cromaticamente legendados de acordo com as áreas de conhecimento. A impressão do modelo, sua divulgação e compartilhamento, entre os CPCS e seus profissionais, propicia a extrapolação do modelo para diferentes contextos em CPCs do país.

O modelo em formato consolidado está disponível para download na íntegra, em https://goo.gl/rVJZN7.

\section{Discussão}

Um modelo de gerenciamento de projetos adaptado para a pesquisa clínica, visando à profissionalização dos centros e à padronização quanto ao gerenciamento, é reconhecido pela literatura pelo sucesso de um projeto, por meio de investimento em técnicas de aplicação de gerenciamento de projetos com objetivo de desperdiçar menos recursos financeiros e atingir os benefícios esperados. São escassos na literatura exemplos de modelos gerencias de centros de pesquisa e, pelo nosso conhecimento, este é o primeiro modelo de gerenciamento de estruturas de pesquisa clínica proposto no cenário brasileiro. 

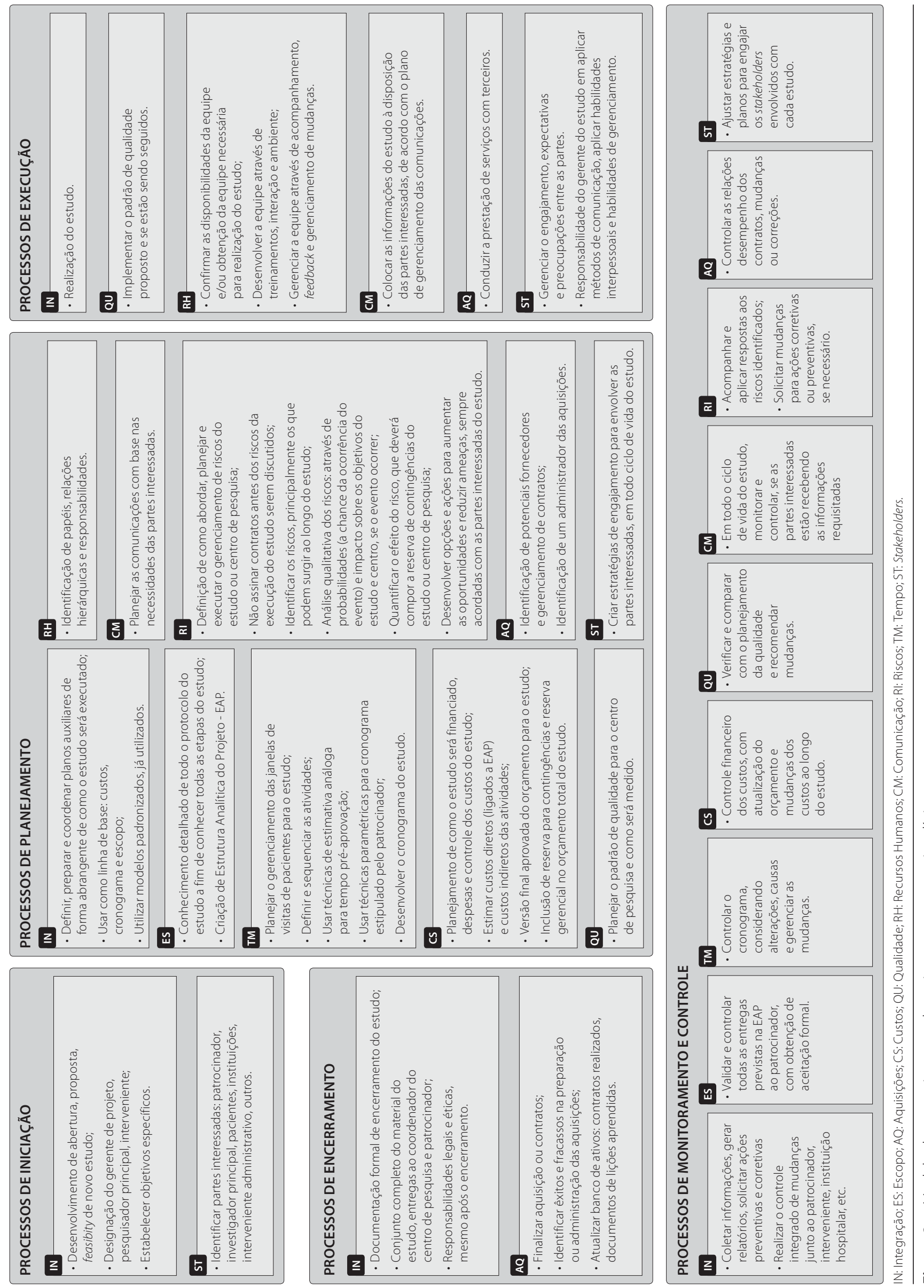
Os principais resultados do estudo demonstram a lacuna no processo de gerenciamento dos CPCs no país, marcada pela carência do profissional com o cargo de gerente de projetos, ausência de gerenciamento financeiro, escasso controle da qualidade do serviço e comunicação deficiente entre as equipes.

Quanto à aplicabilidade de técnicas analíticas para o processo de planejamento e monitoramento e controle dos custos, os centros de pesquisa apresentaram respostas positivas quanto à realização de controle das despesas e controle do faturamento (receitas) dos estudos. Entretanto, no que se refere à rentabilidade, o resultado mais frequente parece ser negativo. Nota-se a diferença entre conhecimento sobre conceitos gerenciais que são relacionados e que influenciam diretamente a estratégia do centro e os resultados econômicos deles.

Para realizar estudos clínicos dentro do orçamento, faz-se necessário planejar, monitorar e controlar os processos de gerenciamento orientados (PMI, 2013; Project Management Institute, 2017). O planejamento orçamentário de um CPC engloba a responsabilidade de analisar previamente os contratos que impactam diretamente os benefícios a longo prazo, atividade designada a um colaborador da própria infraestrutura do CPC. Entretanto, em nosso estudo, entre os colaboradores dos centros de pesquisa respondentes, o profissional com o cargo de gerente de projetos é inexistente em mais da metade dos centros ativos no país. Esse cenário é diferente do apresentado nos EUA e Europa, onde a carreira de gerente de projetos está consolidada (International Network of Research Management Societies, 2017; Society of Research Administrators International - SRA International, 2017). Em estudo realizado no Brasil também se confirmou a carência desse profissional para organizar eficazmente um projeto científico (Monteiro et al., 2014).

No cenário global, o relatório do PMI apontou as principais falhas em iniciativas estratégicas, tais como: falta de objetivos e marcos claramente definidos para medir o progresso, comunicação pobre e falta de comunicação por parte da alta administração (Project Management Institute, 2017). Na rotina dos centros de pesquisa, as falhas de comunicação foram apontadas como inexistentes para a maioria dos participantes da formação desse modelo. Porém, a literatura demonstra que, no setor de projetos, as comunicações são responsáveis por grande parcela no sucesso das empresas. Tal diferença entre os resultados e os dados da literatura provoca incerteza sobre o entendimento da questão pelos respondentes do questionário ou o quanto as ligações entre as partes interessadas são prejudicadas por meio da comunicação.

O volume de estudos clínicos que os centros possuem em andamento refletem no resultado financeiro das partes interessadas. O repasse do êxito do negócio aos pesquisadores é realizado trimestralmente na maior parte dos contratos, conforme os centros participantes dessa pesquisa. Isso demonstra a necessidade de acompanhamento eficaz dos resultados, a fim de garantir o repasse contínuo, de acordo com as entregas realizadas. Um reflexo dessa realidade é a falta de dedicação exclusiva por parte dos integrantes da equipe para com o CPC.

Todo o processo de desenho de um estudo clínico, desde o planejamento até o fechamento, visa à obtenção de resultados com elevada qualidade científica (Tenório et al., 2017). Em artigo publicado em 2014, o qual apresenta uma análise crítica da qualidade dos CPCs na cidade de Belo Horizonte, ficou evidente que, além dos parâmetros de qualidade exigidos pela Instrução Normativa no 4 (Anvisa), faz-se necessária a inserção de novas ferramentas e estratégias para melhor controle dos processos e da qualidade (Lima et al., 2014).

Técnicas e ferramentas da área administrativa e de gerenciamento de projetos vêm sendo incorporadas na busca da melhoria da qualidade. Em um hospital privado de São Paulo, foi implementado um programa de gestão da pesquisa científica a partir da aplicação de ferramentas de gestão empresarial Lean Seis Sigma e PMBOK ${ }^{\circledR}$ no gerenciamento de projetos, porém o resultado não foi propagado de forma generalizada para os centros do país como forma de produto ou de um modelo capaz de ser replicado em diferentes centros de pesquisa (Hors et al., 2012).

Desenvolver novos processos e divulgá-los para os centros do país é uma das formas de melhorar a competitividade do Brasil diante do mercado da pesquisa e inovação (United Nations Educational, 2015). Para serem eficazes, novos processos necessitam de profissionais qualificados e especializados trabalhando na implementação das práticas. Portanto, a profissionalização dos centros de pesquisa cria uma vantagem competitiva, influenciando na seleção dos centros para a realização de novos estudos clínicos.

Também foi possível identificar neste estudo que há um volume de centros de pesquisa que não participam do cadastro e de iniciativas governamentais por meio da Rede Nacional de Pesquisa Clínica (RNPC), mas que poderiam colaborar com os objetivos da rede e fortalecer a pesquisa clínica no país.

A taxa de resposta do questionário demonstra o crescente interesse na área de gestão em pesquisa clínica, o envolvimento dos centros de pesquisa e o interesse no resultado da proposta deste estudo, embora um artigo publicado em 2013 sobre avaliação do nível de maturidade no gerenciamento de projetos em uma instituição de pesquisa clínica tenha demonstrado que os centros ainda não fazem uso rotineiro de metodologias e práticas sistematizadas em gerenciamento de projetos (Bramati, 2013). 
Corroborando essa realidade de carência de iniciativas voltadas a tornar os centros mais organizados e ágeis, a implantação em EGP ainda não é uma realidade para a grande parte dos centros. Um EGP pode ser usado no planejamento estratégico da organização, alinhando-o com os interesses da alta direção (PMl, 2013).

Algumas limitações em nosso modelo merecem ser mencionadas, como as encontradas no uso do questionário on-line, a impossibilidade de auxílio ao participante quando ele não compreende alguma pergunta, assim como o não conhecimento das circunstâncias em que o questionário foi respondido. Entretanto, o benefício do uso desse tipo de ferramenta para a coleta de dados deve ser ainda mais difundido, estudado e aprimorado, por se tratar de uma ferramenta de baixo custo, fácil desenvolvimento, acesso e análise de informações (Faleiros et al., 2016).

\section{Conclusões}

Os resultados do presente estudo revelaram que a aplicação desse modelo nos centros de pesquisa possibilita uma meIhor definição do escopo de cada estudo, de modo que os custos e prazos possuam baixa margem de variabilidade e que seja possível estabelecer boa comunicação entre as partes envolvidas. Como resultado, o impacto econômico do gerenciamento será reconhecido.

Para pesquisas futuras, recomenda-se o acompanhamento da aplicação do modelo de gerenciamento in loco e a comparação dos resultados com a performance prévia do centro de pesquisa. Com o propósito de consolidar as práticas, propõem-se adequação conforme as peculiaridades locais, incluir detalhamento e novas ferramentas recomendadas pelo PMBOK${ }^{\circledast}$, mensurando os benefícios econômicos e estratégicos, ao passo que essas medidas tornem os centros de pesquisa no Brasil mais atrativos e competitivos para a participação de estudos multicêntricos internacionais de relevância para a saúde da população brasileira.

\section{Referências bibliográficas}

Bramati l. Avaliação do nível de maturidade no gerenciamento de projetos em uma instituição de pesquisa clínica. Revista de Gestão e Projetos. 2013.

Faleiros F, Käppler C, Pontes FAR, Silva SSC, Goes FSN, Cucick CD. Use of virtual questionnaire and dissemination as a data collection strategy in scientific studies. Texto Contexto - Enferm. 2016;25(4):2-7.

Hors C, Goldberg AC, Almeida EHP, Babio Júnior FG, Rizzo LV. Aplicação das ferramentas de gestão empresarial Lean Seis Sigma e PMBOK no desenvolvimento de um programa de gestão da pesquisa científica. Einstein (São Paulo). 2012;10(4).

International Network of Research Management Societies. INORMS International Network of Research Management Societies. Disponível em: http://www.inorms.net/index.html. Acesso em: 3 jun. 2017.

Laboratórios Pfizer. Pesquisa Clínica/Laboratórios Pfizer/Saúde para uma vida melhor. Disponível em: http://www.pfizer.com.br/sobre-a-pfizer/ industria-farmaceutica/pesquisa-clínica. Acesso em: 3 jun. 2017.

Lima KS, Vidal BB, Lage APD, Wainstein AJA. Análise crítica da qualidade dos centros de pesquisa clínica de Belo Horizonte. Ciênc Cult. 2014;66(1):34-7.

Monteiro CBM, Almeida Junior DM, Wajnzstejn R. Gerente de projeto na saúde e pesquisas médicas. Rev Bras Crescimento Desenvolv Hum. 2014;24(3):239-42.

PMI. A Guide to the Project Management Body of Knowledge (PMBOK ${ }^{\circledast}$ Guide) - Fifth Edition. Project Management Journal. 2013;44:e1.

Project management institute. Success Rates Rise 2017 9th Global Project Management Survey. PMI's Pulse of the Profession; 2017. p. 32.

Sabbag PY. Criação e Viabilidade de Projetos e Gerenciamento de Projetos: Gerenciamento de Projetos e Empreendedorismo (customizado). 2a ed. São Paulo: Saraiva; 2013.

Silva RE, Amato AA, Guilhem DB, Novaes MRCG. Globalization of clinical trials: ethical and regulatory implications. Int J Clin Trials. 2016;3(1):1-8.

Society of research administrators international - SRA International. SRA International.org. Disponível em: https://www.srainternational.org/. Acesso em: 24 jul. 2017.

Tenório M, Mello GA, Viana AL. Políticas de fomento à ciência, tecnologia e inovação em saúde no Brasil e o lugar da pesquisa clínica. Ciênc Saúde Coletiva. 2017;22(5):1441-54.

United Nations educational, Unesco Science Report: towards 2030 Executive Summary. Disponível em: http://www.unesco.org/openaccess/terms-use-ccbynd-port. Acesso em: 24 jul. 2017. 